\title{
Secondary syphilis presenting with features of cranial arteritis
}

\author{
P. J. T. DREW \\ M.A., M.B., M.R.C.P. \\ Chelmsford District Hospitals, Essex
}

\begin{abstract}
Summary
The case of an elderly man with secondary syphilis is described. The clinical features at the time of presentation were suggestive of cranial arteritis and there was a substantial but incomplete response to corticosteroids. Subsequent treatment with penicillin proved highly effective.
\end{abstract}

\section{Introduction}

Cranial arteritis is now recognized as a fairly common affliction of the elderly, classically characterized by headache, scalp tenderness, and the presence of a raised erythrocyte sedimentation rate (ESR) (Paulley and Hughes, 1960). These clinical findings are by no means pathognomic and can be mimicked by a variety of conditions ranging from local disease of the scalp or skull to lesions of the trigeminal nerve in the brain stem (Walton, 1977).

By contrast, secondary syphilis has now become an uncommon condition and acute syphilitic meningitis is rare (Catterall, 1977). Headache is the commonest symptom of the condition but scalp tenderness is infrequent (Merritt and Moore, 1935). In the case to be presented, these 2 features dominated the clinical picture and led initially to an incorrect diagnosis of cranial arteritis.

\section{Case report}

A 68-year-old single man presented to the Outpatient Department complaining of severe headache of 5 weeks duration and scalp tenderness such that he could not bear to put his head on the pillow at night. Examination revealed tenderness over the right temple and over a prominent temporal artery. His only other symptoms at this stage were occasional feelings of being 'off balance' and a complaint that there had been a slight worsening of long-standing deafness. Neurological examination revealed no abnormality apart from mild bilateral sensorineural hearing loss. The ESR was $72 \mathrm{~mm} / \mathrm{hr}$.

Cranial arteritis was diagnosed and he was started on a therapeutic trial of prednisolone, $\sqrt{y}$ $30 \mathrm{mg} /$ day. Over the next 3 weeks there was con- 9 siderable but incomplete improvement in his $\omega$ symptoms. Mild scalp tenderness persisted but his $\underset{\sim}{\sim}$ ESR fell progressively to $17 \mathrm{~mm} / \mathrm{hr}$. Two weeks after starting treatment a faint non-irritant maculo- $\supset$ papular rash was observed and during the fourth $\vec{z}$ week of treatment he reported a considerable $O$ deterioration with episodes of vertigo and vomiting $\Phi$ and marked hearing loss in the right ear. Headache $\frac{3}{6}$ was no longer a problem but he was mentally $\mathbb{C}$ depressed. Examination revealed that the macule $\overrightarrow{0}$ papular rash was more pronounced and covered ho trunk, all 4 limbs and the soles of his feet. In additio there was complete deafness in the right ear and $\mathrm{a}$. mild sensorineural deafness in the left ear. There was diminished sensation to pin-prick over the territory supplied by the ophthalmic division of the $\frac{\alpha}{\varnothing}$ right trigeminal nerve, with a diminished right $\varrho$ corneal reflex, and an area of hypoaesthesia over the $\overrightarrow{\vec{O}}$ medial aspects of both calves. The pupillary reac- 3 tions and optic fundi were normal.

He was admitted to hospital. Results of standard tests for syphilis in the blood showed VDRL positive $\overline{0}$ (titre of 1 in 32), TPHA positive and FTA (abs) positive. Other investigations showed a normal $\frac{}{3}$ haemoglobin, urea and electrolytes and tests of liver: function. The hepatitis B surface antigen was neg-윽 ative. Proctoscopy revealed 3 painless anal fissures negative by dark ground examination for Treponema $\mathrm{S}$ pallidum and sigmoidscopy was otherwise normal. The cerebrospinal fluid was clear with white cells $88 \times 10^{9} / 1(100 \%$ lymphocytes) and no red cells, $N$ a protein of $0.6 \mathrm{~g} / \mathrm{l}$ and glucose of $3.64 \mathrm{mmol} / \mathrm{l}^{\circ}$ (blood glucose $6 \cdot 16 \mathrm{mmol} / \mathrm{l}$ ). Standard tests for syphilis were positive in the CSF with a VDRL $\omega$ titre of 1 in 8 and a Lange colloidal gold curve of 11233322. A temporal artery biopsy performed after 4 weeks' treatment with steroids showed no inflammation. An electroencephalogram showed very low voltage but was otherwise normal and $\frac{T}{0}$ computerized axial tomography of the brain $\frac{\overrightarrow{\mathbb{D}}}{}$ 
demonstrated mild generalized cerebral atrophy only.

Secondary syphilis with meningovascular involvement was diagnosed and treated with intramuscular procaine penicillin $600000 \mathrm{u}$./day for 21 days. The prednisolone was withdrawn over a 2-week period. Shortly after the first injection of penicillin he developed a transient pyrexia with intense irritation of his rash for the one and only time, features consistent with a mild Jarisch-Herxheimer reaction (Warrell et al., 1971). The rash faded over the next 3 or 4 days. During treatment he complained of left-sided headache and the ESR rose to a peak of $98 \mathrm{~mm} / \mathrm{hr}$. Analgesics were given and the headache resolved a few days after completion of treatment. Four months later he was asymptomatic with an ESR of $22 \mathrm{~mm} / \mathrm{hr}$ and a VDRL test positive with serum diluted 1 in 4 and positive with undilute CSF. The latter was in other respects normal. Serial audiometry documented a modest return of hearing in the right ear, and the other sensory abnormalities all resolved. All attempts to trace a contact were unsuccessful.

\section{Discussion}

Headache in secondary syphilis is usually attributed to meningeal invasion, although in the majority this is clinically silent (Catterall, 1977). In later stages of the disease lightning pains can occur in the face with tabes dorsalis and gummata can form in the skull and meninges and cause persistent symptoms (Merritt, 1973). Headache is a common symptom of general paralysis and is presumably a reflection of the meningeal inflammation present in this condition (Merritt, 1973).

In this case, a specific inflammation of the temporal artery as part of a more widespread vascular inflammation would seem improbable.More likely, inflammation at the base of the brain affected fibres of the trigeminal nerve, causing a neuropathy, initially with increased sensitivity and pain, and subsequently with relative anaesthesia. The eighth nerve was presumably affected by the same process.

A definitive account of syphilitic meningitis from the pre-antibiotic era (Merritt and Moore, 1935) identified 3 overlapping types of presentation. In the first group, the symptoms of headache, nausea and vomiting were associated with signs of meningeal irritation and bilateral papilloedema. Inflammation predominantly affecting the meninges in the posterior fossa and producing obstruction to the flow of cerebrospinal fluid was held responsible and the entity was labelled 'acute syphilitic hydrocephalus'. In the second group, convulsions, pyramidal tract signs and mental disturbances predominated and were attributed to meningitis mainly affecting the vertex. In the third group, the symptoms were associated with the presence of cranial nerve palsies and, less frequently, with signs of meningeal irritation; this was 'meningitis of the basilar type' and the case reported here seems to fit best into this group.

Of the 34 cases of basilar meningitis collected in the Merritt and Moore study, the eighth nerve was involved in 13 cases and the fifth nerve in 7 cases, although in only one of these 7 was sensory function affected alone. The prognosis was good even without penicillin and, as in the case reported here, most of the cranial nerve lesions made a good recovery.

\section{Acknowledgments}

My thanks to Dr Frederick Lees for allowing me to report this case and to Mr D. G. Fife, Dr G. W. Jenkins and Dr P. B. Thavabalan for their help and assistance.

\section{References}

Catterall, R.D. (1977) Neurosyphilis. British Journal of Hospital Medicine, 17, 585.

MERRIT, H.H. (1973) A Textbook of Neurology (5th edn) p. 109. Lea and Febiger, Philadelphia.

MERritT, H.H. \& MoORE, M. (1935) Acute syphilitic meningitis. Medicine, 14, 119.

Paulley, J.W. \& Hughes, J.P. (1960) Giant cell arteritis or arteritis of the aged. British Medical Journal, 2, 1562.

Walton, J.N. (1977) Brain's Diseases of the Nervous System, 8th edn, p. 36. Oxford University Press, Oxford.

Warrell, D.A., Perine, P.L., Bryceson, A.D.M., Parry, E.H.O. \& Pepe, Helen M. (1971) Physiological changes during the Jarisch-Herxheimer reaction in early syphilis. American Journal of Medicine, 51, 176. 